\title{
Effetto dei poliaspartati sulla stabilità tartarica e sul colore dei vini rossi
}

\author{
Antonella Bosso, Massimo Guaita, Silvia Motta, Loretta Panero, Andriani Asproudi, e Maurizio Petrozziello \\ Consiglio per la Ricerca in Agricoltura e l'Analisi dell'Economia Agraria, Centro di Ricerca per l'Enologia di Asti \\ (CRA-ENO), via Pietro Micca, 3514100 Asti, Italia
}

\begin{abstract}
Sintesi. La stabilizzazione tartarica dei vini può essere ottenuta asportando una parte del potassio e/o l'acido tartarico presenti o con l'aggiunta di additivi (acido metatartarico, gomme di cellulosa, mannoproteine) in grado di impedire la formazione e/o l'accrescimento dei cristalli di bitartrato di potassio, principale responsabile della comparsa di precipitati in bottiglia. Il presente lavoro ha riguardato lo studio dell'efficacia stabilizzante di nuove molecole: i poliaspartati (PASPs) su vini rossi. Lo studio aveva, inoltre lo scopo di verificare se, dopo l'aggiunta o durante la conservazione in bottiglia, l'apporto di queste molecole determini intorbidamento e/o perdita di colore nei vini rossi. I risultati evidenziano che i PASPs possiedono interessanti proprietà stabilizzanti nei confronti delle precipitazioni tartariche, simili a quelle dell'acido metatartarico. La presenza di PASP nei vini rossi non ha determinato perdite di antociani totali o modificazioni dei parametri cromatici (intensità e tonalità) né subito dopo l'aggiunta, né durante la conservazione. In un solo vino analizzato, subito dopo l'aggiunta di PASP (controllo dopo 48 ore), si è osservato un leggero aumento della torbidità nella tesi trattata. L'effettuazione di una chiarifica con bentonite $(40 \mathrm{~g} / \mathrm{hL})$ prima dell'aggiunta del PASP ha consentito di evitare l'intorbidamento del vino.
\end{abstract}

\section{Introduzione}

La stabilizzazione tartarica dei vini può essere ottenuta asportando il sale di KHT in eccesso per precipitazione a bassa temperatura [1], allontanando una parte degli ioni bitartrato e potassio per elettrodialisi [2] o gli ioni potassio con l'impiego di resine scambiatrici di cationi. Nelle fasi che precedono l'imbottigliamento sono talvolta impiegati additivi con proprietà stabilizzanti nei confronti della precipitazioni di bitartrato di potassio. Nella UE è autorizzato l'impiego di acido metatartarico (MTA), carbossimetilcellulosa e mannoproteine [3-6].

Recenti ricerche [7] hanno verificato che i poliaspartati (PASPs) [8] possiedono proprietà stabilizzanti simili a quelle del MTA, ma sono molto più stabili nel tempo. Per queste loro caratteristiche i PASPs potrebbero essere impiegati come additivi per la stabilizzazione tartarica dei vini. La presente ricerca si è occupata di approfondire alcuni aspetti del comportamento di queste molecole quanto aggiunte a vini rossi. In particolare, lo studio ha indagato l'effetto sulla stabilità tartarica, del colore e colloidale di vini rossi subito dopo l'aggiunta e dopo un anno di conservazione in bottiglia.

\section{Materiali e metodi}

La prima esperienza ha riguardato il confronto tra 4 PASPs - A-3C: sale di sodio e peso molecolare medio (PM) pari a $3 \mathrm{KDa}$; A-5DK: sale di potassio e PM pari a $5 \mathrm{KDa}$; C-5D: sale di sodio e PM pari a $5 \mathrm{KDa}$; $\mathrm{C}-$ 10D: sale di sodio e PM pari a $10 \mathrm{KDa}$ ed un MTA, tutti aggiunti alla dose di $100 \mathrm{mg} / \mathrm{L}$ ad un vino rosso Dolcetto della vendemmia 2012. Dopo l'aggiunta dei prodotti i vini sono stati imbottigliati e conservati in una camera termostatata alla temperatura di $20^{\circ} \mathrm{C}$. I controlli analitici sono stati effettuati dopo 48 ore dalle aggiunte e dopo 1 anno di conservazione in bottiglia. E' stata valutata la stabilità tartarica dei vini con l'impiego del test a freddo, la torbidità, il contenuto in antociani e flavonoidi totali ed il colore (A420+A520 e A420/A520).

La seconda esperienza è stata effettuata su un vino rosso Syrah della vendemmia 2013. Il vino è stato suddiviso in 3 aliquote: testimone, chiarificata con gelatina $(15 \mathrm{~g} / \mathrm{hL})$ e chiarificata con proteina vegetale di pisello $(30 \mathrm{~g} / \mathrm{hL})$. Ciascuna di queste 3 tesi è stata quindi suddivisa in 2 aliquote: una testimone ed una trattata con bentonite $(40 \mathrm{~g} / \mathrm{hL})$. Metà del volume di ciascuna tesi è stato quindi aggiunto del PASP A-5D (sale di potassio con PM pari a $5 \mathrm{kDa}$ ) alla dose di $100 \mathrm{mg} / \mathrm{L}$. Dopo 48 ore dall'aggiunta e dopo 1 anno di conservazione in bottiglia sui vini è stata determinata la torbidità, il tenore in antociani totali e flavonoidi totali e il colore (A420+A520 e A420/A520).

Il test a freddo consisteva nella misurazione della variazione del tenore in acido tartarico del vino per HPLC [9] prima e dopo permanenza a bassa temperatura $\left(-4{ }^{\circ} \mathrm{C}\right)$ per 6 giorni [5]. La determinazione del tenore in antociani e flavonoidi totali è stata effettuata per spettrofotometria [10].

\section{Risultati}

Prima esperienza su vino Dolcetto. In figura 1 sono riportati i risultati del test a freddo condotto sui vini dopo 48 ore dall'aggiunta dei PASPs e del MTA. Si osserva che tutti i vini aggiunti di PASPs e MTA sono stabili nei confronti delle precipitazioni tartariche $\left(\Delta \mathrm{H}_{2} \mathrm{~T} \approx 0 \mathrm{~g} / \mathrm{L}\right)$, mentre soltanto $\mathrm{i}$ vini testimone risultano instabili. 


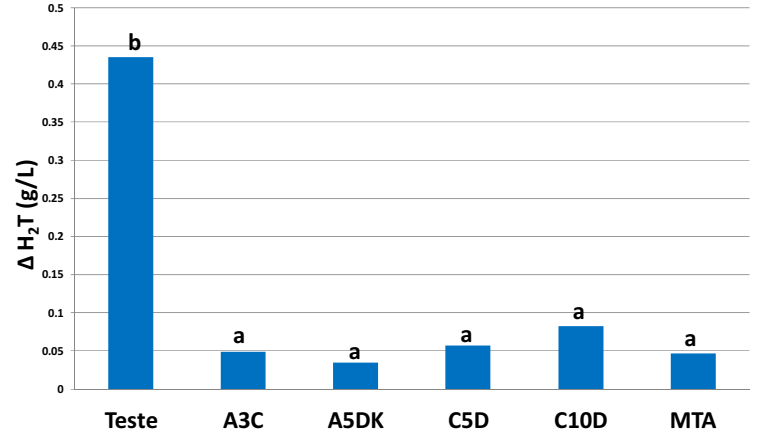

Figura 1. Risultati del test a freddo dopo 48 ore dall'aggiunta degli additivi.

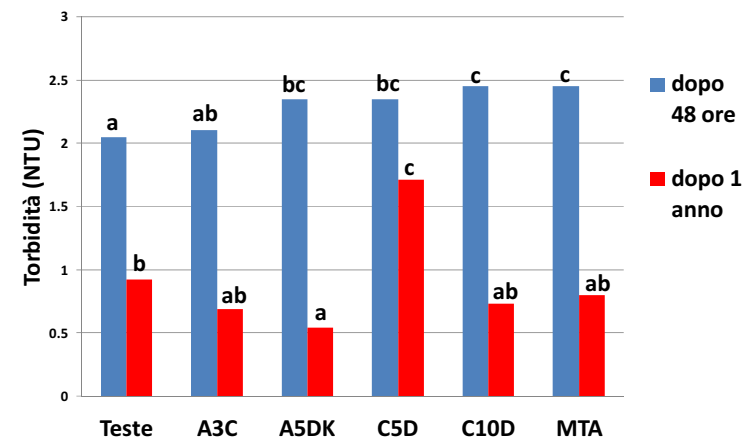

Figura 2. Torbidità misurata dopo 48 ore e dopo 1 anno dalla aggiunta degli additivi.

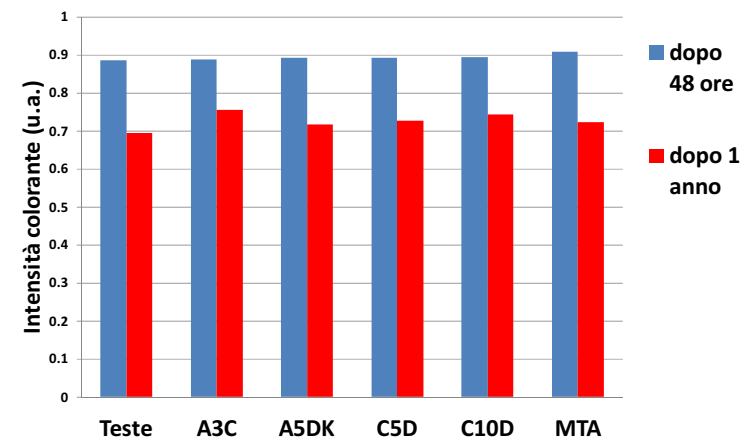

Figura 3. Intensità colorante misurata dopo 48 ore e dopo 1 anno dall'aggiunta degli additivi.

Dopo l'aggiunta dei prodotti non si osservano modificazioni di rilievo della torbidità (Fig. 2), cambiamenti del colore (A420+A520 e A420/A520) (Figs. 3 e 4) o perdite di antociani e flavonoidi totali (Figs. 5 e 6), malgrado per alcuni di questi parametri le differenze risultino statisticamente significative tra alcune tesi.

Nel corso della conservazione del vino in bottiglia il colore evolve, l'intensità colorante diminuisce e la tonalità aumenta, ed il contenuto in antociani e flavonoidi totali e la torbidità si riducono. Dopo 12 mesi di bottiglia non si rilevano differenze di rilievo tra le tesi per la torbidità (Fig. 2), il colore (Figs. 3 e 4) e il contenuto in antociani e flavonoidi totali (Figs. 5 e 6 ).

Seconda esperienza. In un precedente lavoro [7] abbiamo rilevato, analizzando un campione di 15 vini rossi prelevati in vasca da alcune cantine private, che l'aggiunta di poliaspartato non ha provocato variazioni del tenore

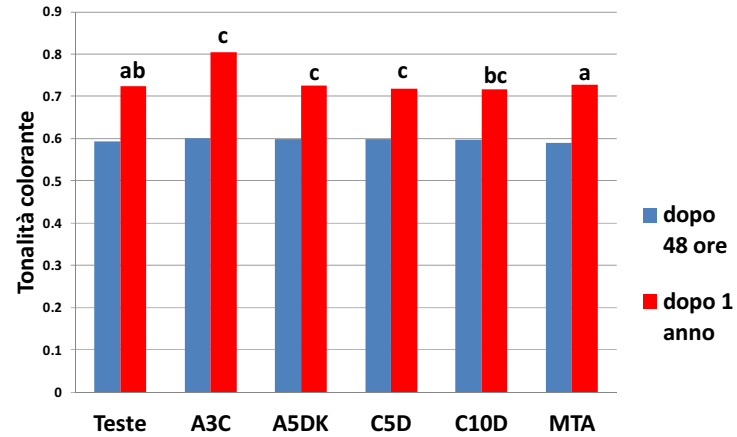

Figura 4. Tonalità del colore misurata dopo 48 ore e dopo 1 anno dall'aggiunta degli additivi.

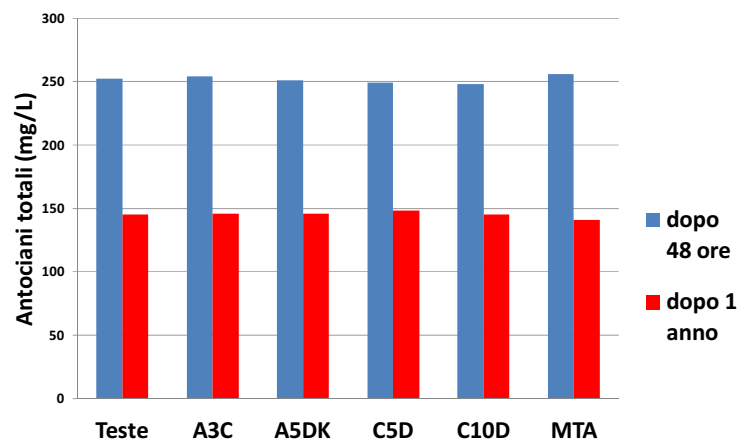

Figura 5. Antociani totali misurati dopo 48 ore e dopo 1 anno dall'aggiunta degli additivi.

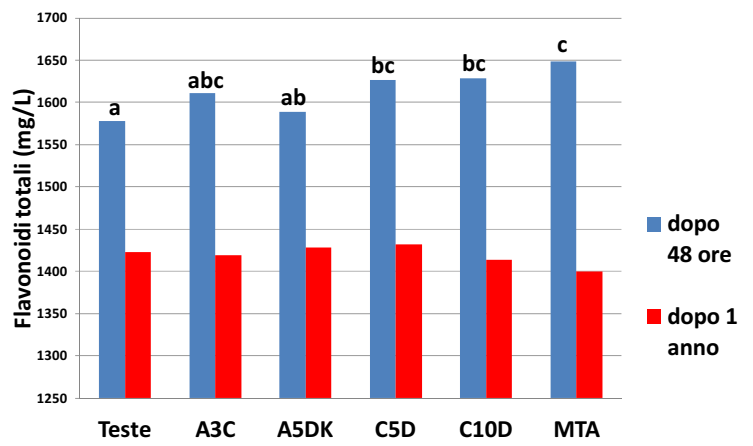

Figura 6. Flavonoidi totali misurati dopo 48 ore e dopo 1 anno dall'aggiunta degli additivi.

in antociani e flavonoidi totali e del colore. Il confronto tra $i$ vini ha successivamente riguardato la valutazione di altri parametri, tra cui la torbidità prima e dopo l'aggiunta del PASP. In un caso, un vino Syrah della vendemmia 2013, dopo aggiunta del PASP A-5D abbiamo osservato un aumento della torbidità.

La tesi testimone di questo vino è stata quindi sottoposta ad una prova consistente nell'effettuazione di un trattamento chiarificante con gelatina o proteina vegetale in abbinamento o meno alla bentonite. I vini delle diverse tesi sono stati quindi addizionati o meno di PASPK $(100 \mathrm{mg} / \mathrm{L})$. In figura 7 sono riportati i valori della torbidità, determinati 48 ore dopo l'aggiunta del PASPK, delle tesi testimoni trattate e non con bentonite ed aggiunte o meno di PASP.

Le tesi trattate con bentonite sono risultate più limpide dei corrispondenti testimoni e si sono mantenute tali dopo 


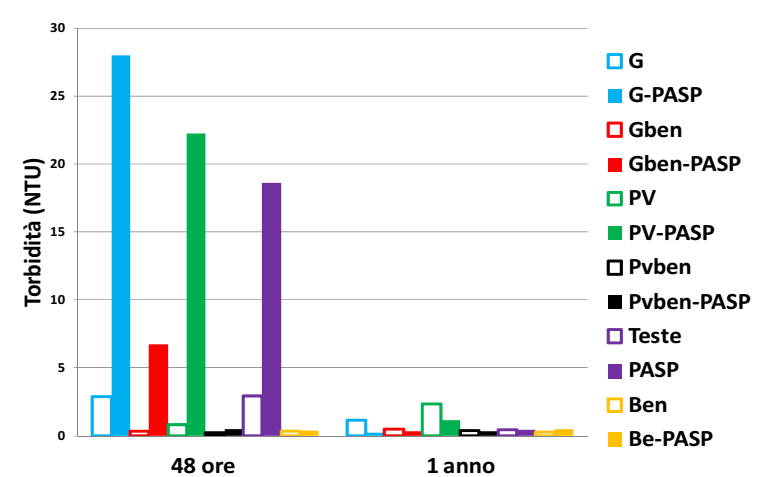

Figura 7. Torbidità misurata dopo 48 ore e dopo 1 anno dalla aggiunta degli additivi.

l'aggiunta del PASPK. Per quanto riguarda le tesi aggiunte di gelatina o di proteine vegetali, il solo trattamento chiarificante con gelatina o proteina vegetale non ha modificato la risposta del vino all'aggiunta di PASPK: si è osservato un aumento della torbidità dopo aggiunta del prodotto. In presenza di un trattamento combinato con bentonite la tesi trattata con proteine vegetali non ha incrementato la torbidità in presenza di PASP, mentre quella chiarificata con gelatina mostra comunque un aumento della torbidità anche se molto più contenuto rispetto al corrispondente testimone senza bentonite. La comparsa di torbido è avvenuta rapidamente, subito dopo l'aggiunta del PASP e la torbidità non è ulteriormente aumentata nel tempo. Nel corso della conservazione i vini si sono progressivamente illimpiditi e un deposito si è formato nelle tesi che si erano precedentemente intorbidate. Dopo un anno di conservazione tutti vini risultano limpidi (Fig. 7). Infine, l'aggiunta di PASP non ha provocato alcuna modificazione del colore dei vini delle diverse tesi (intensità e tonalità cromatica) sia dopo 48 ore dall'aggiunta che dopo un anno di conservazione in bottiglia (Fig. 9). Lo stesso andamento è stato osservato per il contenuto in antociani e in flavonoidi totali (dati non riportati).

\section{Discussione e conclusioni}

I risultati raccolti nel corso della presente esperienza confermano quanto già rilevato in un precedente lavoro [7] e cioè che $\mathrm{i}$ poliaspartati sono molecole in grado di impedire la precipitazione tartarica in vini instabili. Il loro effetto subito dopo l'aggiunta al vino risulta simile a quello del MTA, ma a differenza del MTA esso si mantiene molto più stabile nel tempo [7]. Per quanto riguarda le interazioni con la materia colorante dei vini rossi, non è stato osservato alcun effetto dell'aggiunta del PASP sul colore (intensità e tonalità cromatica) e sul tenore in antociani e flavonoidi totali, né sulla loro evoluzione durante la conservazione ( 1 anno di bottiglia). In un solo caso (vino Syrah della vendemmia 2013) si è verificato un aumento della torbidità del vino dopo l'aggiunta del PASP. Una chiarifica preliminare del vino con l'impiego di bentonite $(40 \mathrm{~g} / \mathrm{hL})$ ha consentito di evitarne l'intorbidamento in presenza di PASP.

Il lavoro è stato finanziato dal Progetto Europeo "STABIWINE Use of biopolymers for sustainable stabilization of quality wines"- Grant Agreement n 314903 (Seventh Framework Programme.Theme: SME-2012-2. Research for SME associations).

\section{Bibliografia}

[1] P. Ribéreau-Gayon, Y. Glories, A. Maujean e D. Dubourdieu, Handbook of Enology, Chichester, UK John Wiley\& Sons, Ltd (2006)

[2] M. Moutounet e J.L. Escudier, Austr. Grapegrower \&Winemaker, Adelaide (1991)

[3] M. Moutounet, J.L. Battle, B. Saint-Pierre e J.L. Escudier, 6ème Symposium d'Oenologie, Paris Lavoisier: 531-534 (1999)

[4] J.C. Crachereau, N. Gabas, J. Blouin, B. Hebrard e A. Maujean, Bull. OIV, 841/842, 151-159 (2001)

[5] S. Motta, A. Bollito, M. Guaita, M. Petrozziello, L. Panero, A. Bosso, Bull. OIV, 941/943, 367-377 (2009)

[6] H. Claus, S. Tenzer, M. Sobe, M. Schlander, H. König, J. Fröhlich, Austr J Grape Wine Res, 20, 186-193 (2014)

[7] A. Bosso, L. Panero, M. Petrozziello, M. Sollazzo, A. Asproudi, S. Motta, M. Guaita, Food Chemistry, doi: http://dx.doi.org/10.1016/j.foodchem. 2015.03 .099 (2015)

[8] A. Steinbüchel, S.R. Fahnestock, Biopolymers, Wiley-VCH (2003)

[9] P. Cane, Enotecnico, 26, 69-72 (1990)

[10] R. Di Stefano, M.C. Cravero, N. Gentilini, Enotecnico, 25, 83-89 (1989) 